\title{
Experiencias y retos del uso de datos de aplicaciones móviles para la movilidad urbana
}

\author{
Experiences and challenges of using mobile application data for urban mobility
}

\author{
Anna-Beatriz Sabino \\ GSD Plus (Colombia) \\ Pedro Reis-Martins \\ Centro de Operações do Rio de Janeiro-COR (Brasil) \\ Mauricio Carranza-Infante \\ Centro de Inteligencia y Operación Semafórica de Medellín (CIOS) (Colombia)
}

Sabino, A. B., Reis-Martins, P., \& Carranza-Infante, M. (2020). Experiencias y retos del uso de datos de aplicaciones móviles para la movilidad urbana. Revista de Arquitectura (Bogotá), 22(I). 82-93. https://doi. org/l0.14718/RevArq.2020.3039

\author{
Anna-Beatriz Sabino \\ Internacionalista, Universidade Federal Fluminense (Brasil). \\ Magíster en planeación urbana y regional, Universidad de los Andes (Colombia). \\ Consultora en proyectos de transporte y movilidad urbana en GSD Plus. \\ (iD https://orcid.org/0000-0002-7556-826 I \\ annabsoliveira@hotmail.com \\ Pedro Reis-Martins \\ Profesional de comunicación social, Pontifícia Universidade Católica, Rio de \\ Janeiro (Brasil) \\ Profesional en gestión de proyectos, Instituto Brasileiro de Mercado de Capitais \\ (IBMEC) (Brasil) \\ Coordinador de planeación del Centro de Operaciones de Rio de Janeiro. \\ (D) https://orcid.org/0000-0003-4633-27/6 \\ preismartins@gmail.com \\ Mauricio Carranza-Infante \\ Ingeniero electrónico, Universidad Francisco José Caldas (Colombia). \\ Vinculado al Centro de Ingeniería y Operación Semafórica (CIOS), Secretaría \\ de Movilidad, Medellín. \\ (D) https://orcid.org/0000-0002-6565-405 I \\ areacarranza@gmail.com
}

\section{Resumen}

La planeación de la movilidad urbana requiere el uso de datos masivos para apoyar la toma de decisiones y realizar proyecciones estratégicas: aun así, muchos gobiernos locales no poseen la capacidad para generar los datos necesarios al respecto; sin embargo, empresas privadas, como Waze, Moovit, Stava y Uber (gestores de aplicativos de movilidad), tienen la capacidad para producir estos datos, $y$, además, han demostrado su disponibilidad para compartirlos y así mejorar las condiciones de la planeación de la movilidad en las ciudades. En América del Sur, ciudades como Río de Janeiro, São Paulo y Medellín, son casos de urbes que se convirtieron en ejemplos de innovación en el de uso de datos. Con base en la experiencia de dichas ciudades y en encuestas aplicadas con representantes de empresas gestoras de apps de movilidad y de gobiernos, en el presente artículo se propone un modelo de tres niveles para el uso de datos en beneficio de la gestión y la planeación de la movilidad urbana. El modelo propuesto tiene como objetivo trazar parámetros que ayuden a las ciudades a desarrollar una visión en cuanto al potencial de los datos para generar acciones y políticas públicas de movilidad urbana.

Palabras clave: apps de movilidad; big data; gestión de tráfico colaborativo; movilidad inteligente; planificación del tránsito; planificación urbana; Smart Cities; transporte

\begin{abstract}
Urban mobility planning is included in a global scenario of increasing use of massive data to support decision making. However, many local governments do not have a structure that produces the data necessary to base their strategic projections. At the same time, private companies - such as Waze and Moovit (mobile application mobility managers) - have the ability to produce this data and, in addition, have demonstrated their availability to share them and thus improve planning conditions in cities. Nevertheless, managing this data and using it for the benefit of better urban planning and management is not a simple task. In South America, Rio de Janeiro, Sao Paulo and Medellín have overcome important obstacles in this trajectory and became examples of innovation in the use of data. Based on the experience of these cities - and on surveys conducted with representatives of mobile apps companies and governments -, this article proposes a three-level model for the use of data for the benefit of urban mobility management and planning. The proposed model is in its initial stage and aims to draw parameters that help cities to develop a vision regarding the potential of data to generate actions and public policies of urban mobility.
\end{abstract}

Keywords: mobility apps; big data; collaborative traffic management; Smart mobility; traffic planning; urban planification; transport; Smart Cities

Recibido: septiembre 9/2019

\section{Introducción}

La planeación de la movilidad urbana es una ciencia dependiente en grado sumo de datos. Tradicionalmente, el sector público es el principal encargado de generar y recopilar información cuantitativa de origen y destino, medios de transporte utilizados, duración y hábitos de transporte; esto, por medio de encuestas domiciliarias de movilidad, o de aforos, que parten de una muestra aleatoria para hacer estimaciones. En el caso de las encuestas domiciliarias, Osorio Arjona y García Palomares (2017) afirman que su complejidad requiere una muestra de datos considerable, lo cual implica un alto trabajo de campo y de procesamiento de la información, lo cual suele culminar en altos costos.

A lo largo de los últimos años, las nuevas tecnologías se presentan como posibles soluciones para superar la dificultad de generación de datos. El sector privado ha ejercido un rol protagónico en este sentido, por medio del acervo de información generada por los usuarios de aplicaciones o por los dispositivos móviles. Esta gran cantidad de información espacial que puede ser capturada, comunicada, agregada, almacenada y analizada (Manyika et al., 2011) —denominada big data- posee, además, otras características: la accesibilidad, la georreferencia y la generación de datos en tiempo real, con alta frecuencia y de fácil actualización (Osorio Arjona \& García Palomares, 2017). 
Con base en estos conceptos, en el presente artículo se busca destacar el potencial de aplicación de estos datos para la gestión y la planeación de la movilidad urbana y para las políticas públicas. Así, se tuvo en cuenta una segunda definición de big data, que se refiere a "las fuentes de datos con diferentes características, cuyas señales débiles no estructuradas de las redes sociales ganan importancia, lo que lleva a diferentes usos posibles de cada fuente de datos en el soporte de políticas públicas" (Azzone, 2018, p. 117).

De esta forma, el término big data gana cada vez más espacio en las discusiones de planeación urbana y políticas públicas. Prueba de ello es la reciente publicación del ITDP Brasil (2019), que reúne los resultados de las discusiones acerca del uso de datos en diversas ciudades brasileñas. El documento revela que los principales desafíos operacionales para la utilización de datos en la planeación y la gestión de la movilidad urbana son la carencia de datos públicos, la falta de recursos para contratar nuevas encuestas y la privación de acceso a los datos de terceros.

Lo que se plantea en el presente artículo va más allá del tema de acceso a los datos generados por aplicaciones móviles (en adelante, aplicaciones o apps) y se enfoca en las formas de utilizarlos en el día a día de las ciudades. En este sentido, el tipo de dato generado tiene un papel fundamental y va de la mano con el modelo de negocio de la empresa que los produce.

Waze $^{1}$, como ejemplo de lo anterior, indica las rutas menos congestionadas entre dos puntos. Además de datos de origen y destino, la empresa consolida datos de velocidad promedio en las vías, los accidentes y eventos diversos. Uber ${ }^{2}$ ofrece el servicio de transporte de pasajeros; entre los datos generados en tal contexto están: el origen y destino de los pasajeros, los puntos de mayor y de menor demanda y los precios pagos por viaje. Moovit ${ }^{3}$ indica opciones de transporte público y genera información de origen y destino de pasajeros, lo que posibilita identificar las zonas y las estaciones de mayor demanda de transporte. Aunque con objetivos distintos, todas estas aplicaciones generan datos masivos con base en la información de sus usuarios. Recientemente, Uber y Waze crearon mecanismos facilitadores para compartir datos con los gobiernos locales, la academia u otros usuarios.

Aunque el proceso de construcción de alianza entre empresas y gobierno locales sea creciente, es importante entender las dificultades enfrentadas para identificar puntos frágiles que necesitan ser primoreados. El reporte del ITDP Brasil (2019) revela algunas de ellas: la negociación entre el poder público y los privados, la dificultad para comprender la perspectiva del negocio

\footnotetext{
Waze Mobile Ltd. («Waze»).

2 Uber Technologies, Inc. ("Uber").

3 Moovit Inc. ("Moovit")
}

del privado y para percibir el valor agregado que estos datos poseen tanto para las empresas como para el poder público.

Tomando en cuenta este escenario de mayor disponibilidad de datos para planear y gestionar la movilidad urbana - pero con una serie de dificultades para ser vencidas-, este artículo tiene tres objetivos principales. Primero, traer a la luz las soluciones y los retos que han sido enfrentados por gobiernos locales y aplicaciones de movilidad urbana en el proceso de apertura y utilización de datos masivos. El segundo objetivo es ejemplificar cómo estos datos han sido utilizados en la gestión y la operación de la movilidad urbana, a partir de tres experiencias en Sudamérica. Finalmente, el último objetivo es proponer un modelo estratégico para asesorar a las ciudades a utilizar los datos recibidos de forma operacional, táctica y estratégica, con el fin de aportar a la movilidad urbana y a las políticas públicas.

\section{Metodología}

La experiencia de Río de Janeiro y Medellín en la construcción de alianzas y el uso de datos masivos fueron las bases para alcanzar el primer y el segundo objetivos de este artículo. En Río de Janeiro, Brasil, el Centro de Operações de la Alcaldía de Río (COR) es pionero en construir alianzas estratégicas con entidades privadas a fin de generar valor para la operación y la gestión de la ciudad. A lo largo de sus diez años de funcionamiento, y con alianzas con Waze (desde 2013) y Moovit (desde 2014), el COR avanza en la utilización de tecnología para beneficio de los ciudadanos. En Medellín, Colombia, los datos de Waze son utilizados por el Centro de Ingeniería y Operación Semafórica (CIOS) para coordinar los semáforos de la ciudad.

El tercer objetivo se apoyó ampliamente en la experiencia de ambas ciudades y fue complementado por las encuestas realizadas con puntos focales de gobiernos locales y de empresas de apps de movilidad. Las labores de investigación y las entrevistas que son la base de este artículo se desarrollaron entre julio y septiembre de 2019.

El primer paso para construir este artículo fue la percepción de la importancia del tema por parte de sus autores. Esta percepción, en el campo metodológico, puede ser identificada como observación participante: los individuos que observan el fenómeno al mismo tiempo participan de ello. Este método permite un mayor acercamiento al fenómeno observado, debido a que el investigador se halla inmerso en la situación o el grupo de estudio, lo que le confiere una condición privilegiada para observar el fenómeno (Mónico et al., 2017, p. 727). En tal sentido, los autores de este artículo describen y cuestionan sus propias experiencias en cuanto a las alianzas entre gobiernos y aplicaciones y el uso de datos para la movilidad urbana. 
(V) Figura 1. Gráfico de tránsito (7) del COR.

Fuente: elaboración propia (2018).

(7) Figura 2. Panel de (1) notificaciones del COR. Fuente: elaboración propia (2018).
Teniendo clara esta concepción, se hace otra aclaración en cuanto a la condición de los autores del presente artículo: dos de ellos intervienen como participante-observador, ya que estuvieron directamente involucrados en la construcción de las alianzas entre el COR (Río) y el CIOS (Medellín) con aplicaciones de servicios de movilidad. Estos autores tuvieron un rol fundamental en la descripción de las experiencias de sus ciudades y aportaron significativamente en la identificación de los elementos que deberían componer las encuestas desarrolladas para el estudio. El tercer autor participó en condición de observador para comparar las experiencias de las ciudades, dibujar y analizar las respuestas a las encuestas y aportar directamente a los modelos propuestos de uso de datos para la planeación de la movilidad y las políticas públicas.

Se elaboraron encuestas para complementar la percepción de los participantes-observadores. La primera, con un total de seis preguntas, fue dirigida a representantes de gobiernos locales que estuvieron involucrados en la formación de alianzas o en el manejo de los datos compartidos (Anexo I. Encuesta aplicada a representantes de gobiernos locales). Dicha encuesta buscó profundizar la percepción de los agentes sobre la ciudad y disminuir algún grado de parcialidad que, por ventura, estuviese presente en la visión de los observadores-participantes. La segunda, con cuatro preguntas, buscó acercarse a la percepción de las empresas
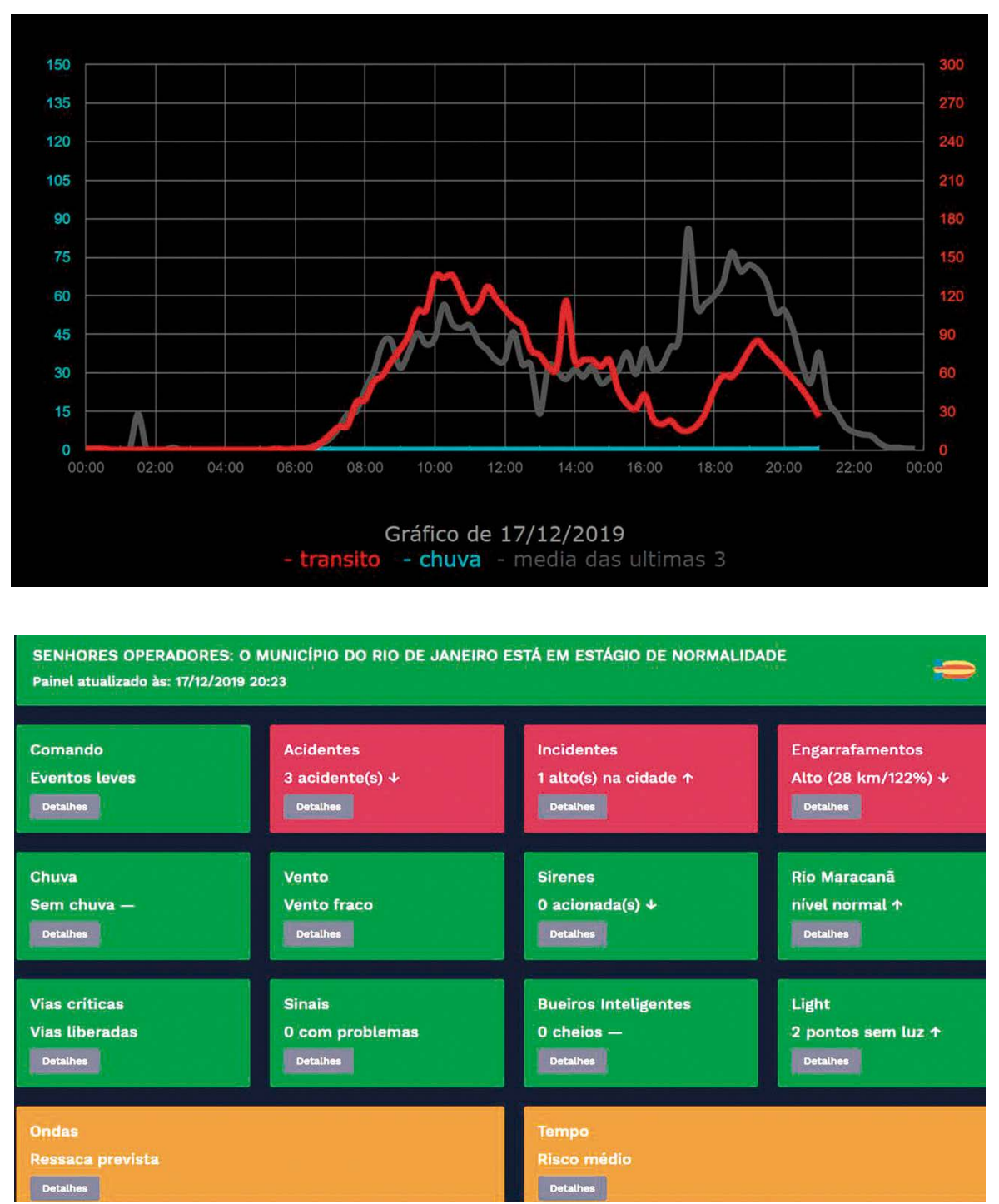

y a entender sus expectativas en cuanto a la creación de alianzas con gobiernos locales (Anexo II. Encuesta aplicada a representantes de empresas).

\section{Resultados}

\section{Experiencias del uso de datos para la gestión operacional y planeación urbana}

\section{Experiencia en Río de Janeiro, Brasil}

Con cerca de 6,7 millones de habitantes (IBGE), Río de Janeiro tiene en el COR su centro municipal de comando y control urbano, como un ambiente de integración entre las agencias de la ciudad que participan en las operaciones de infraestructura, logística y emergencias de la ciudad. Junto al COR, Waze (2013) implementó una de sus primeras alianzas directas con gobiernos locales en el mundo, con el objetivo inicial de facilitar la gestión de las operaciones para la visita del papa Francisco a Río, durante la Jornada Mundial de la Juventud.

Los primeros productos de la colaboración entre el COR y Waze fueron el uso de datos para la gestión operacional urbana en tiempo real y la planeación de restricciones viales de corto plazo. En el caso de la visita del papa Francisco, los cierres de calles previstos fueron programados en el mapa de Waze. Los cierres imprevistos también fueron comunicados en la aplicación por un profesional de Waze posicionado en la sala de control del COR. Inicialmente, la inserción de esta información fue realizada manualmente; posteriormente, se desarrolló una integración tecnológica (API), lo que permitió automatizar el proceso.

Entre 2015 y 2017, se produjeron otras funcionalidades a partir de estos datos de Waze, aún dedicadas a la gestión operacional. Una de ellas fueron los reportes enviados por usuarios de la aplicación, que pasaron a figurar en el mapa situacional del COR. Esto generó una nueva fuente de datos sobre problemas de movilidad y permitió que el equipo del COR identificara más rápido los accidentes y otras anormalidades viales en áreas donde no existían cámaras ni sensores.

Con los datos referidos, el COR desarrolló un mapa de calor para indicar las zonas con más alto índice de tránsito lento. Además de eso, los datos de Waze pasaron a alimentar otras herramientas utilizadas en el COR. Una de ellas es el gráfico de tránsito que apunta en tiempo real el sumatorio de congestionamientos de la ciudad y lo compara con las condiciones de movilidad vial esperadas (mediante un promedio calculado con datos de las tres últimas semanas). En la figura 1, la línea roja es el congestionamiento monitoreado en tiempo real, y la gris, el que se esperaba; los números en rojo indican la cantidad total de kilómetros de atascos en Río. Estos datos pueden ser monitoreados en el videowall de la sala de control; los gestores operacionales pueden accederlos, incluso, por teléfono móvil. 
La alianza entre el COR y Waze también permitió alertar a los usuarios de la aplicación sobre imprevistos en la ciudad, como accidentes de alto impacto y problemas de seguridad pública. En casos más extremos, cierres viales fueron indicados en el mapa digital del Waze y los conductores fueron desviados, de manera automática, a rutas más seguras. Adicionalmente, la compañía local de tránsito (CET-Río) empezó a archivar los datos de Waze para utilizarlos en acciones de planeación de tránsito como, por ejemplo, analizar la eficacia de los cambios de planeación para grandes eventos en el estadio Maracaná.

A partir de 2018, el COR desarrolló un panel para unificar los datos del Waze con los provenientes de sensores instalados en la ciudad y generar alertas sobre riesgos u ocurrencias en la ciudad (figura 2). Cuando hay más de un reporte de accidente vial, por ejemplo, y una calle registra un comportamiento anormal de velocidad promedio - comparada con los datos históricos-, la combinación de estos parámetros emite una alerta de riesgo (amarillo para riesgo mediano y rojo para alto riesgo), que es inmediatamente investigada por el equipo del COR.

El panel de notificaciones del COR apunta para una nova fase de desarrollo de herramientas ambicionadas por el COR, utilizando los datos de movilidad: funcionalidades con uso inteligencia artificial, análisis de datos y big data.

\section{Hallazgos de la experiencia del COR}

Los principales desafíos enfrentados por el COR para concretar alianzas con empresas privadas $y$ utilizar sus datos masivos se detallan en la figura 3.

Actualmente, el principal reto del COR es habilitar los softwares utilizados en la gestión urbana para que recomienden, de forma proactiva y automática, las acciones predeterminadas por los procesos operacionales. Para ello, el COR está trabajando en la identificación de situaciones clave, en la definición y la programación de parámetros básicos, alineados con un gran volumen de datos. Esta labor permitirá generar dos tipos de recomendaciones: para el equipo del COR, acciones alineadas con los procesos de respuestas a incidentes y emergencias urbanas, a partir de los sistemas tecnológicos; para los ciudadanos, mejores rutas u opciones de transporte público, con base en los datos de comportamiento diario de los usuarios (Waze, Moovit y sistemas de transporte público). Los desafíos actuales del COR se muestran en la figura 4 .

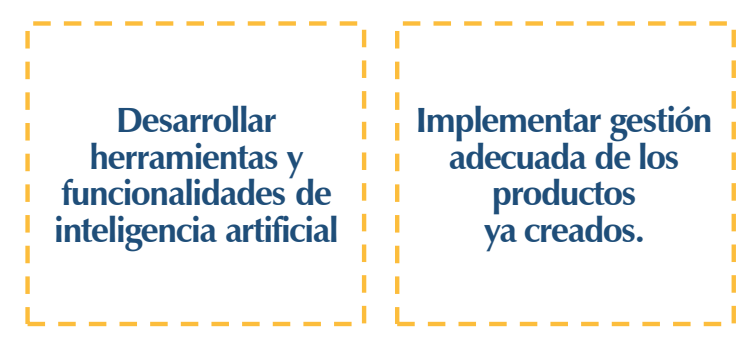

\section{Experiencia en Medellín (Colombia)}

Con sus 2,5 millones de habitantes (DNP, 2019), la capital antioqueña es también un centro generador y atractor de viajes. Las 689 intersecciones semafóricas y los 429 controladores semafóricos de la ciudad son monitoreados desde el CIOS. Desde 2016, el equipo de ingenieros de tráfico del CIOS adoptó una estrategia innovadora para tratar la congestión vial: distribuir el tráfico vial de la ciudad con base en el diseño, el rediseño y los cambios semafóricos con base en los datos de Waze.

El equipo del CIOS hizo estudios para evaluar el impacto de los cambios en el plan semafórico sobre la velocidad de las vías y la relación de dichos cambios con los datos posteriormente emitidos por Waze. A partir del método de vehículo flotante (Vargas \& Sánchez, 2002, p. $8)^{4}$, se pudo percibir que al utilizar los datos de Waze para ajustar la programación semafórica se alcanzaron ahorros de tiempo en el recorrido; ello, debido a los mayores tiempos de luz verde en áreas congestionadas y a la disminución de la longitud de la cola de vehículos detenidos. Los ahorros de tiempo ocasionados por la nueva

4 El método del vehículo flotante permite la toma de información en un tramo de vía de variables - como velocidad, longitud de cola, congestión, nivel de saturación-, mediante el uso de un vehículo que hace de forma repetitiva el tránsito sobre el trayecto de la vía objeto de estudio.

(1) Figura 4. Desafíos actuales del COR.

Fuente: elaboración propia (2019).

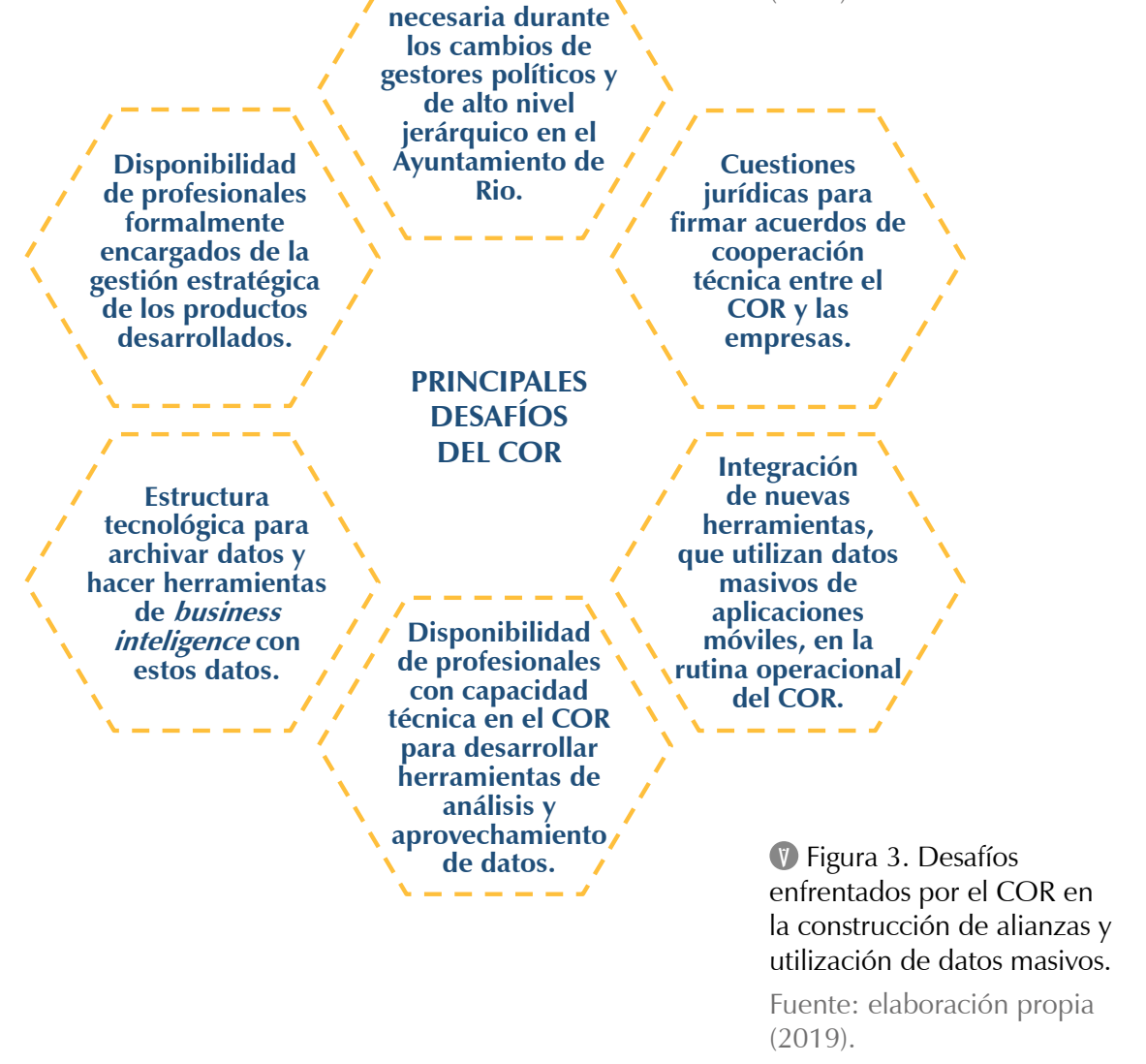
Entrenar el equipo $\quad$ para planear del COR y asegurar que la utilización de las herramientas creadas para la rutina operacional.

Utilizar los datos
para planear
intervenciones
estratégicas,
elaborar y
monitorear políticas
públicas de
movilidad urbana

Utilizar datos de estos las aplicaciones en iniciativas de innovación abierta 


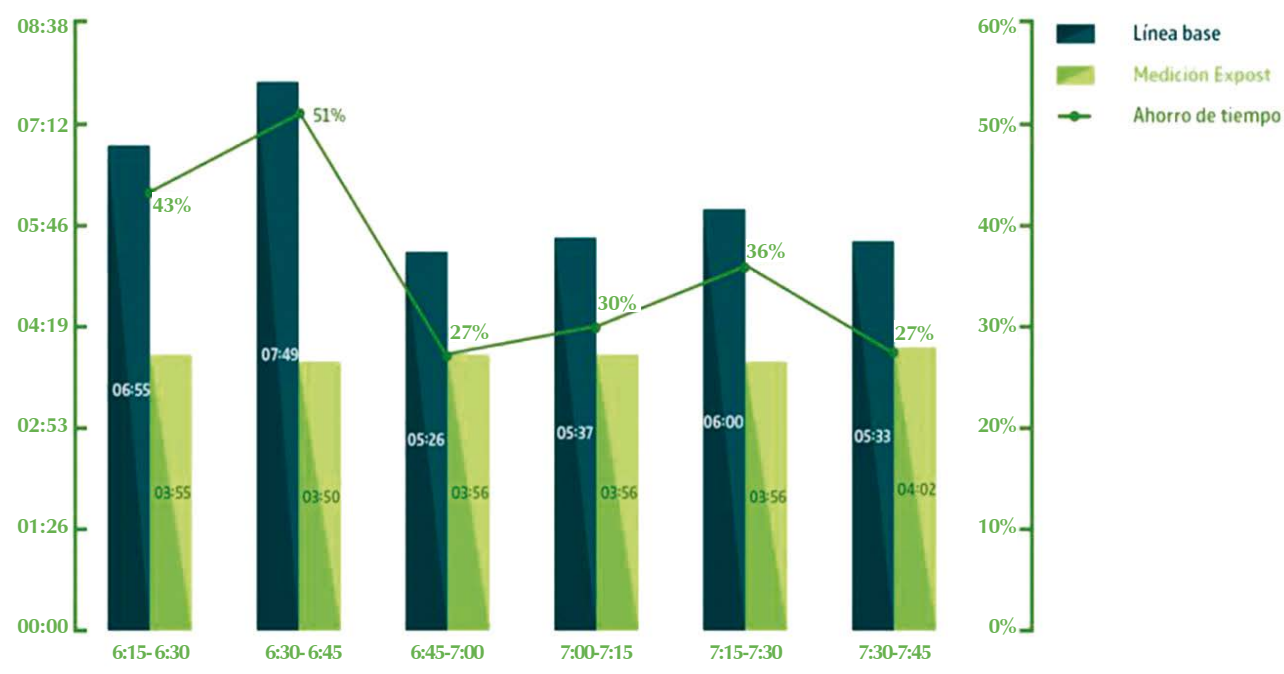

FACILITADORES DEL CIOS

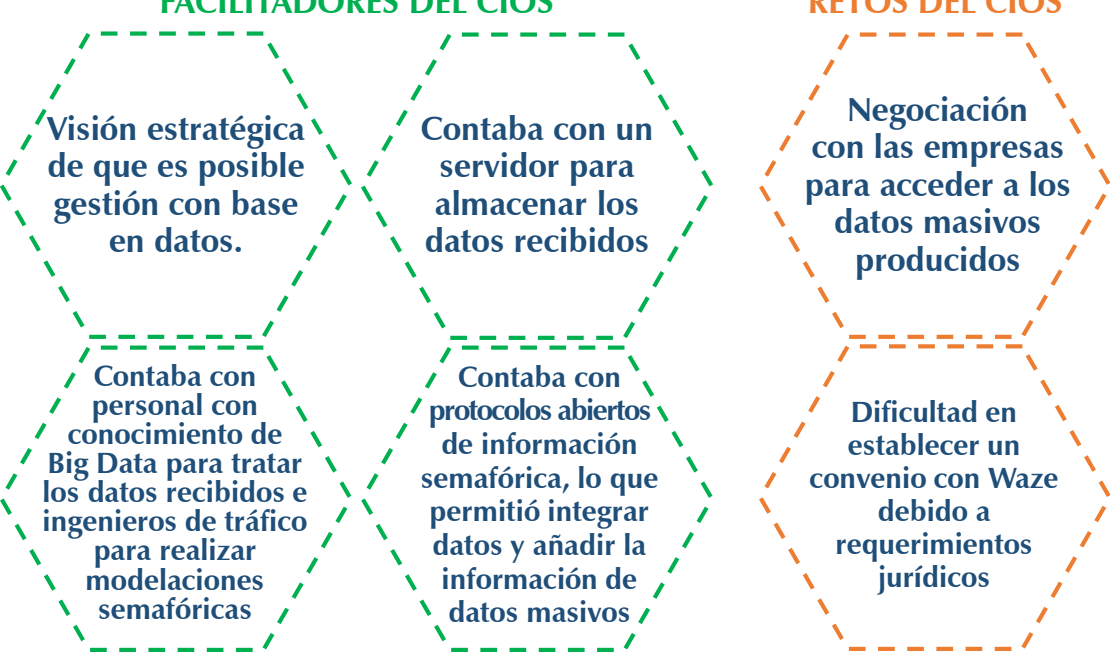

(4) Figura 5. Tiempos de (A) recorrido por periodo (Corredor Av. San Juan w-e/a.m)

Fuente: elaboración propia (2019).

(A) Figura 6. Facilitadores y retos (A) de la experiencia del CIOS Fuente: elaboración propia (2019). estrategia llegaron al 51\% entre las 6:30 a. m. y las 6:45 a. m., como se evidencia en la figura 5 .

El estudio concluyó que cerca de doce minutos después del reajuste semafórico, los indicadores de Waze presentaban disminución de la congestión. El paso siguiente fue desarrollar una plataforma capaz de recibir los datos de Waze para reconocer las vías congestionadas y hacer, automáticamente, el cambio semafórico. Hoy día, este procedimiento es replicado en diez corredores de la ciudad. El CIOS está trabajando en la integración con otros centros de gestión (Metro y corredor semaforizado del tranvía, Metroplus y sistema de prioridad del corredor Metroplus, TPM gestión y control de flota) para mejorar las condiciones de movilidad de la ciudad (Latin America Regional Summit Waze Día 2).

Los datos de Waze permitieron cerrar una carencia que tenía la ciudad: la falta de sensores en vías. En este sentido, la utilización de datos masivos de apps se convierte en una solución de bajo costo de implementación y mantenimiento, a la vez que permite tener un gran número de sensores, que funcionan de forma simultánea e indican comportamientos de movilidad (Carranza et al., 2018). Como contrapartida, la ciudad ofrece información de primera mano sobre cierres viales, obras y eventos para mantener actualizada la red de Waze.

\section{Hallazgos de la experiencia del $\mathrm{CIOS}$}

Previamente al acercamiento a Waze, el CIOS buscó alianzas con Strava ${ }^{5}$, Moovit y Google ${ }^{6}$ para acceder a los datos y verificar los comportamientos de movilidad. De todos los intentos, la alianza con Waze fue la que funcionó, debido a las facilidades tecnológicas y de reducción de costos generadas a partir del programa de la compañía, llamado Connected Citizens Program ${ }^{7}$, dedicado a fomentar alianzas de Waze con ciudades. La experiencia previa de Waze con Río de Janeiro derivó en un modelo de convenio internacional que, para Medellín, no resultó funcional. Las dificultades enfrentadas en la formalización del convenio llevaron a una flexibilización de sus requisitos por parte de Waze. Hoy día, las ciudades interesadas deben diligenciar un formato, abrir una cuenta en Waze y aceptar las condiciones de uso de la plataforma. Eso demuestra que la construcción de alianzas con Medellín y Río de Janeiro dejó lecciones aprendidas, tuvo sus peculiaridades y por eso debe ser mejor comprendida. Los elementos diferenciales de la experiencia de Medellín están resumidos en la figura 6.

La labor ejecutada en el CIOS garantizó el apoyo del Banco Interamericano de Desarrollo (BID) para validar el modelo utilizado en la gestión semafórica. El objetivo del BID es desarrollar estudios para verificar la posibilidad de implementar el modelo en otras ciudades, lo que permitiría reducir el costo de implementación de sensores en las vías y generar nuevos modelos de gestión semafórica que requieran menos recursos humanos.

\section{Experiencia en São Paulo (Brasil)}

La ciudad de São Paulo innovó en la utilización de datos masivos para beneficiar la movilidad activa. La alianza con Strava —una aplicación utilizada por ciclistas para registrar los tramos recogidos - permitió a la ciudad comprender mejor el uso de la bicicleta y mejorar la planeación para construir ciclorrutas. En São Paulo, la aplicación cuenta con cerca de 90.000 usuarios (Avelleda, 2018). Los mapas de calor generados por Strava son utilizados para evidenciar la demanda de espacio público por parte de los ciclistas y para visualizar las rutas más utilizadas. Avelleda (2018) afirma que la información de Strava es de gran utilidad para dar visibilidad al ciclista, comprobar y convencer a la población de la importancia de mantener ciclorrutas en determinadas vías donde se creía que el flujo de ciclistas era irrelevante. La figura 7 muestra el mapa de calor generado en Strava.

Además del uso práctico de los datos recibidos, São Paulo dio un paso importante respecto al reconocimiento de la importancia estratégica

\footnotetext{
5 Strava, Inc. ("Strava")

6 Google LLC ("Google").

7 Sitio del programa en la web disponible en https://www. waze.com/pt-BR/ccp
} 
de dichos datos. El Decreto 58.595 de 2019 establece reglas y condiciones para la operación de empresas de tecnología de servicios para la movilidad y determina que estas deben compartir los datos necesarios para el control y la regulación de políticas públicas de movilidad urbana con la Secretaria Municipal de Mobilidade e Transportes (SMT), de acuerdo con un formato y una periodicidad definidos por el Poder Público (Decreto No. 58.595 de 2019). El referido decreto es una demostración de que el tema tiene una importancia estratégica y que está inserido en la agenda pública paulista: no solo se busca reglamentar los servicios prestados, sino también, garantizar el acceso a los datos masivos producidos.

\section{Resultados de las encuestas aplicadas}

En total, fueron computadas doce respuestas para las encuestas: nueve respuestas vinieron de representantes de gobiernos locales de Río de Janeiro y Medellín; otras tres, de representantes de Moovit y Google. Los principales resultados preliminares de esta encuesta son resumidos a continuación.

\section{Respuestas de gobiernos locales}

Para los representantes de gobiernos locales, fueron presentadas preguntas sobre: el grado de interés y las motivaciones para la utilización de datos generados por las apps de movilidad urbana; los principales retos para concretar alianzas con las empresas propietarias de estas apps; las dificultades para, efectivamente, recibir los datos, y acerca de las necesidades de inversión financiera para dichos proyectos. Las encuestas aplicadas revelaron preliminarmente que las ciudades tienen gran interés en recibir los datos de las aplicaciones, con el $100 \%$ de los respondientes señalando nivel "alto" o "muy alto" de interés.

A partir de una escala de 0 a 5, donde 0 significa "poca relevancia", y 5, "muy relevante", la encuesta también identificó las principales motivaciones de los representantes de ciudades para acceder a estos datos. "Formular, acompañar y monitorear políticas públicas" y "apoyar la gestión operacional de la movilidad urbana" fueron los dos principales motivos, con puntuaciones de 4,4 y 4,0, respectivamente. En relación con los retos enfrentados para establecer alianzas con empresas de apps, "los acuerdos jurídicos requeridos o las normativas específicas del sector público" fueron lo más relevante, con una valoración de 3,9. "La ausencia de consenso acerca de las responsabilidades de inversión financiera que resultan de la alianza" fue el segundo reto considerado más relevante, con de 3,6. El tercer reto considerado relevante (con puntuación de 3,2) fue la "dificultad para convencer a las empresas dueñas de datos de los beneficios de alianzas con las ciudades", lo cual indica que, tal como ocurre con algunos ejemplos, como los de Waze y Uber, posiblemente aún es necesa-

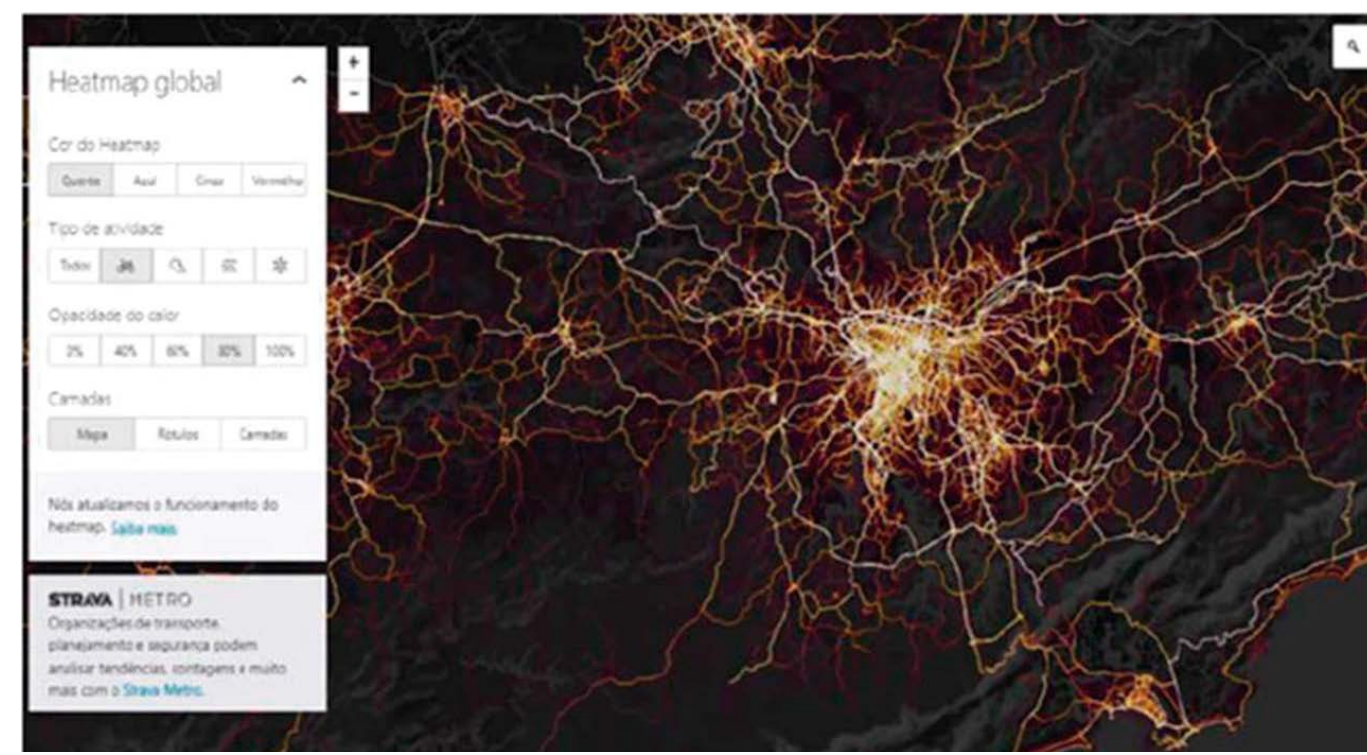

rio expandir en el sector privado el concepto de (A) Figura 7. Mapa de calor de colaboración público-privada con uso de datos Strava. para mejorar la calidad de la gestión y la planea- Fuente: Avelleda (2018). ción de la movilidad urbana.

Finalmente, el reto apuntado como más significativo (por parte de la capacidad de los gobiernos) para la utilización de los datos compartidos por empresas fue "la indisponibilidad de recursos financieros por parte de la ciudad para destinar a las actividades relacionadas al compartimiento de datos" (con puntuación de 3,6), seguida por otros dos retos, que obtuvieron, cada uno, puntuación de 3,1: "carencia de recursos y personal calificado" y "ausencia de herramientas tecnológicas por parte de la ciudad".

\section{Respuestas de empresas privadas}

La encuesta aplicada a los representantes de empresas de apps de movilidad urbana contenía preguntas sobre el grado de interés de la empresa en abrir datos para los gobiernos locales; los objetivos de las compañías al abrir dichos datos; los principales retos para concretar alianzas con ciudades y las dificultades encontradas para, efectivamente, compartir datos. En una escala de 0 a 5, donde 0 es "muy bajo", y 5, "muy alto" (interés en compartir dados con ciudades), las respuestas revelaron una puntuación de 3,7, lo que, de forma preliminar, puede ser considerado interés relevante.

Así como en la encuesta para gobiernos locales, fue utilizada también una escala de 0 a 5 para respuestas, en la cual 0 significa "poco relevante", y 5, "muy relevante". Las respuestas a las encuestas permitieron identificar, preliminarmente, que los principales intereses de las empresas son: "ofrecer herramientas y apoyar la gestión operacional de la movilidad urbana en las ciudades" y "crear una buena relación con gobiernos locales" —las 2, con puntuación de 5; o sea que el 100\% de los respondientes indicaron estas 2 opciones como las más relevantes-. Por otro lado, las respuestas sobre los principales retos para concretar la alianza con gobiernos locales señalaron en primer lugar los "acuerdos jurídicos requeridos o 
las normas específicas del sector público" (con calificación de 5 puntos), seguidos por la "dificultad para convencer a los gobiernos locales de los beneficios de la alianza planteada por la empresa" (con 4,7 puntos). La "carencia de personal calificado, por parte de los gobiernos" es la principal dificultad al momento de, efectivamente, compartir los datos con las entidades públicas, con valoración de 4 puntos.

La figura 9 resume los resultados encontrados con la aplicación de las encuestas para cada tipo de actor.

\section{Discusión}

\section{Enfoques propuestos para el uso de datos de apps de movilidad en la gestión de ciudades}

Los objetivos del presente artículo van de la mano con la estrategia de ciudad inteligente, definida por Rodríguez como:

\section{(...) una serie de iniciativas, políticas y acciones cuyo objetivo prioritario es favorecer la movilidad en las ciudades de modo que ésta no suponga una traba al quehacer diario en las urbes ni a su desa- rrollo. Se trata de una serie de actuaciones que lo que pretenden es facilitar la movilidad de los usuarios bien sea a pie, en bicicleta, transporte público o privado, bajo una premisa común: aho- rro en costes económicos, ambientales y tiempo (Rodríguez, 2013).}

Para Sandí Meza, el camino hacia el modelo de ciudades inteligentes requiere transversalidad y multidisciplinariedad en las estrategias de gobierno, y la incorporación de tecnología para políticas públicas más abiertas, participativas, transparentes y sostenibles. Haciendo énfasis en la relación entre entidades públicas y privadas, el autor afirma que las ciudades inteligentes promocionan el conocimiento tecnológico del sector privado y las necesidades de que dispone el sector público (2017, p. 27).

Las experiencias detalladas en el presente estudio son una evidencia de la necesidad del enfoque estratégico para maximizar las posibilidades de éxito de los proyectos colaborativos entre los sectores públicos y privados. Los casos y los resultados de las encuestas presentados contienen elementos que aportan al establecimiento de alianzas entre gobiernos locales y empresas de apps, con el fin de desarrollar proyectos colaborativos que resulten en sistemas de movilidad más eficientes a partir del uso de datos masivos.

A fin de identificar estrategias eficaces para la concretización de alianzas, fueron mapeadas las variables que influencian su desarrollo y los actores clave en este proceso. La experiencia de Río de Janeiro y Medellín permitió realizar un ejercicio preliminar de caracterización de las formas de uso de los datos masivos por parte de los gobiernos locales. Este ejercicio se encuentra en su etapa inicial, pero permitió clasificar las inicia- tivas de uso de datos en tres niveles: operacional, táctico y estratégico.

Los niveles de análisis de proyectos de alianzas entre gobiernos locales y empresas de apps de movilidad

El primer nivel de uso de datos, denominado operacional, se caracteriza porque busca responder a eventos en tiempo real y mejorar la gestión operacional de la ciudad. Los datos son utilizados a diario para identificar la ocurrencia de eventos específicos que, sin una respuesta adecuada e inmediata, tienden a impactar de manera directa en el funcionamiento de la ciudad, la provisión de servicios públicos y la movilidad urbana. Por lo general, las acciones realizadas siguen protocolos predefinidos y una estructura de toma de decisiones de baja complejidad. Ejemplos de uso de datos en un nivel de análisis operacional son la gestión semafórica en Medellín y las respuestas y las acciones del COR en Río de Janeiro.

El nivel de análisis táctico busca planear acciones para reducir el impacto de eventos previsibles; por lo tanto, tiene una naturaleza de planeación operacional. El tipo de intervención que será planeado y el involucramiento institucional requerido dependerán del evento al que se busca responder. En este sentido, los datos masivos sirven para revisar patrones históricos de movilidad de los puntos de la ciudad que serán impactados por el evento planeado. Ejemplos de uso de datos en el contexto táctico son la planeación de operaciones especiales de tránsito — cierres viales, eventos diversos, regreso a las clases y gestión del tránsito durante los festivos-.

El nivel de análisis estratégico busca utilizar los datos de forma analítica o preventiva. En este sentido, los datos son utilizados para comprender un fenómeno o para planear o justificar una acción de mediano o largo plazo y de mayor amplitud espacial en la ciudad. Para convertir los datos en información, así como a fin de hacer un análisis más profundizado y útil para la toma de decisiones de alto nivel, la muestra de datos debe ser más amplia que la requerida para el nivel de análisis táctico, lo que demanda más tiempo y recursos humanos y tecnológicos. La acción de la alcaldía de São Paulo en cuanto al mantenimiento de las ciclorrutas en vías con mayor flujo de ciclistas es un ejemplo de uso de datos masivos con fines estratégicos de mediano o largo plazo. La figura 8 resume las características de los niveles operacional, táctico y estratégico en cuanto al tipo de datos requeridos, a la acción que va a llevarse a cabo y su objetivo final.

Obstáculos preliminarmente identificados en las encuestas

Además de los niveles de alcance del uso de datos, las encuestas hechas en el presente estudio permitieron apuntar las motivaciones 

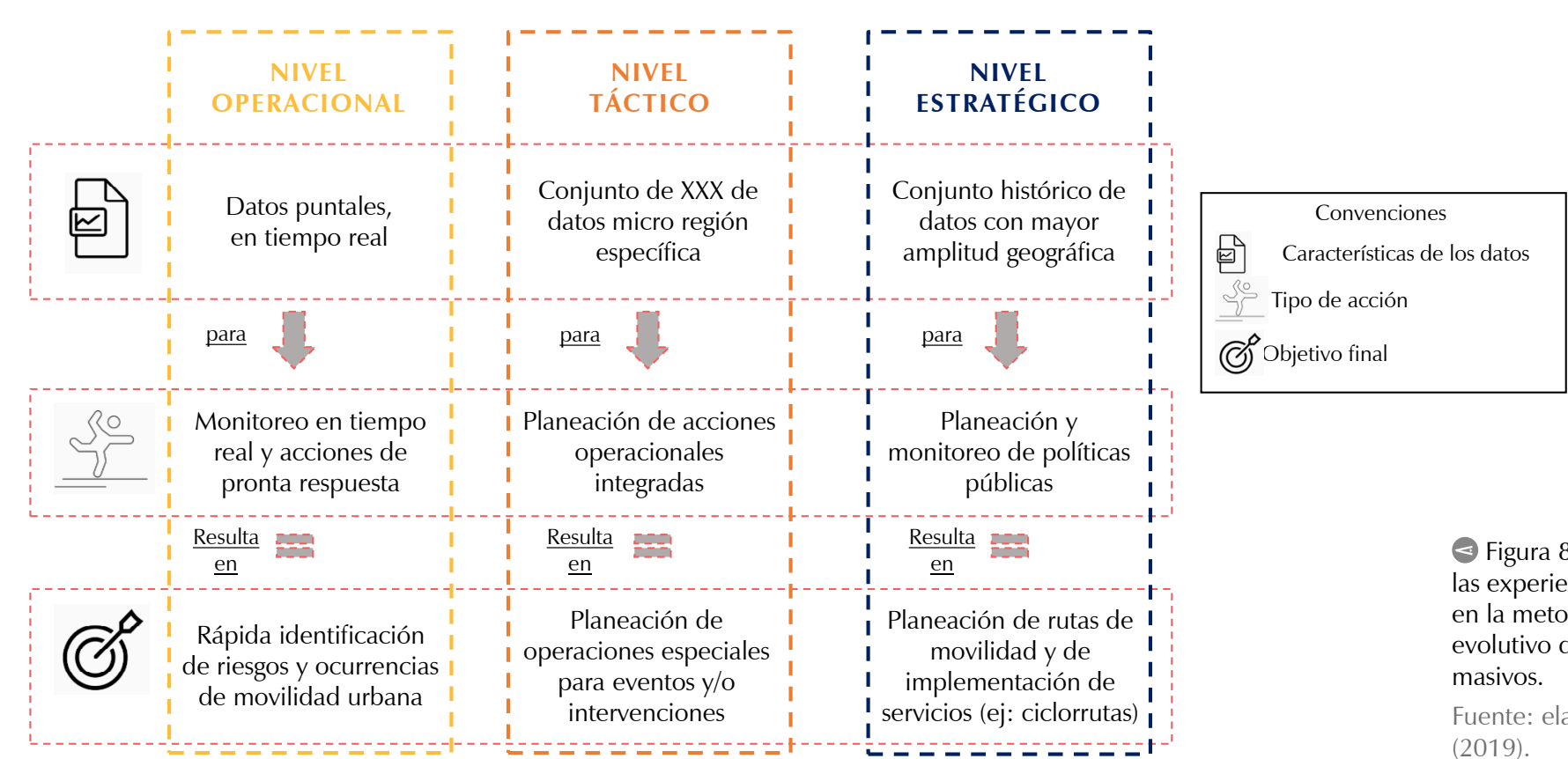

y las dificultades para la implementación de alianzas entre gobiernos locales y empresas de aplicaciones. La figura 9 resume los resultados encontrados.

Como se puede apreciar en la figura 9, los resultados de la encuesta revelan temas comunes entre gobiernos y empresas. El interés en mejorar la gestión operacional de las ciudades y las dificultades presentadas por los acuerdos jurídicos fueron apuntados por ambos actores. En Río de Janeiro y Medellín, por ejemplo, las reglas jurídico-burocráticas de la administración pública retardaron temporalmente la formalización de las alianzas con Waze. Facilitar acuerdos jurídicos de esta naturaleza sería una medida relevante para fomentar estos proyectos de movilidad inteligente.

La carencia de profesionales de tecnología calificados también fue de importancia en ambas ciudades. Aunque las alcaldías cuenten con un cuerpo de profesionales, estos no están preparados para analizar los datos recibidos. Por lo tanto, la contratación de profesionales calificados debe ser priorizada por las ciudades que tienen la ambición de tornarse más inteligentes. En el caso de Río, formar alianzas con universidades locales es una estrategia utilizada como intento para remediar dicho problema. Sobre la ausencia de recursos financieros, este es un desafío continuo de las ciudades latinoamericanas. Se requiere seguir investigando el tema para comprender cómo otras ciudades han ultrapasado los obstáculos enunciados y cuáles son las mejores prácticas y caminos posibles para su solución.

Por otra parte, según la encuesta, la sinergia entre gobiernos y empresas se cuestiona cuando los gobiernos locales afirman tener alto interés en establecer las alianzas, pero las empresas de apps destacan la baja participación de los gobiernos como un reto para consolidar las alianzas. Este es otro elemento que reafirma la necesidad de seguir investigando el tema y la posición de ambos actores.

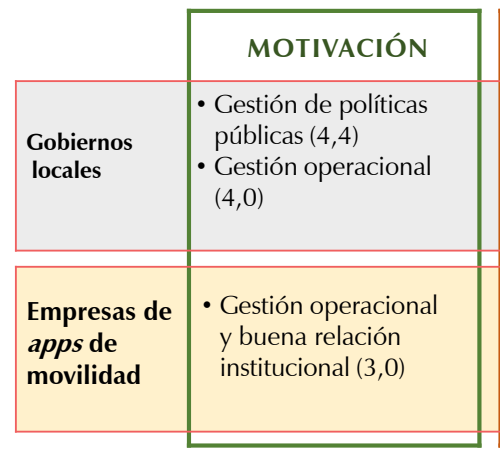

\begin{tabular}{|l||}
\hline \multicolumn{1}{|c|}{ RETOS DE LA } \\
IMPLEMENTACIÓN \\
\hline - Acuerdos jurídicos $(3,9)$ \\
- Ausencia de recursos \\
financieros $(3,7)$ \\
- Ausencia de herramientas \\
tecnológicas $(3,1)$ \\
\hline - Acuerdos jurídicos $(5,0)$ \\
- Baja participación de \\
los gobiernos $(4,7)$ \\
- Ausencia de personal \\
calificado $(4,0)$
\end{tabular}

\begin{tabular}{|l|}
\hline \multicolumn{1}{|c|}{ CONCLUSIONES } \\
\hline $\begin{array}{l}\text { - Gobiernos locales } \\
\text { muestran alto interés } \\
(4,7 / 5,0) \text { en establecer } \\
\text { alianzas. }\end{array}$ \\
\hline - Empresas muestran menor \\
interés $(3,7 / 5,0)$ en \\
establecer alianzas.
\end{tabular}

Los resultados expuestos en el presente trabajo sirvieron de base para la construcción de una propuesta de modelo evolutivo de uso de datos de aplicaciones móviles, que puede ser utilizado como referencia por cualquier ciudad. Este modelo, todavía en desarrollo, busca traer a la luz elementos básicos para que gobiernos locales empiecen a desarrollar sus propios proyectos a partir de los datos disponibles.

El objetivo principal del modelo presentado es servir como referencia para que los municipios comprendan el potencial del uso de datos para la gestión de la movilidad. A partir de conceptos básicos, cada ciudad podrá preparar su propio ambiente, de acuerdo con las necesidades, los recursos y los arreglos institucionales que posee, y llegar así a una "ruta segura" propia para proyectos con uso de datos de apps de movilidad. Para esto, los gobiernos locales deben comprender los esfuerzos requeridos para desarrollar iniciativas en los tres niveles presentados. Al mismo tiempo, deben ser conscientes de las expectativas de las empresas de aplicaciones de movilidad en relación con las alianzas y el valor añadido que tiene la información que poseen.

Es importante destacar que el potencial de uso de datos no debe limitarse al nivel operacional, pero sí tener un enfoque de largo plazo para incrementar la capacidad de resiliencia de las ciudades. La planeación de la respuesta a emergencias y la planeación operacional de la ciudad
(4) Figura 8. Clasificación de las experiencias observadas en la metodología de modelo evolutivo de uso de datos masivos.

Fuente: elaboración propia (2019). 
en casos de eventos de alto impacto son formas de sacar mayor provecho de la infraestructura disponible y de gestionar mejor los eventuales impactos logísticos.

La visión presentada en seguida sobre cómo empezar con iniciativas más sencillas y progresar hacia proyectos complejos es un punto de partida para discusiones sobre el tema, a partir de casos de éxito en Latinoamérica. Se destaca que los casos de estudiados, aunque son exitosos, se mantienen en los niveles operacional y táctico, sin significar hasta la fecha una significativa contribución para el desarrollo de políticas públicas; sin embargo, los resultados obtenidos por dichas ciudades contribuirán a crear un ambiente más favorable para que datos de apps de movilidad sean efectivamente insumos para crear, revisar o evaluar políticas públicas en un futuro no tan lejano. Por medio de buenas estrategias de uso de datos, las ciudades podrán saltar del uso operacional y táctico a niveles más estratégicos, que permitirán revisar, monitorear, e, incluso, elaborar, políticas públicas capaces de mejorar la calidad de los servicios de movilidad urbana.

El modelo evolutivo de uso de datos y sus características

Los niveles de análisis presentados (operacional, táctico y estratégico) son la base del modelo preliminar planteado que sugiere una ruta para los gobiernos locales hacia el uso de datos masivos de apps de movilidad con el fin de generar, progresivamente, impactos positivos para la gestión urbana; ello, porque, si bien parece claro el potencial de los datos para primorear las políticas públicas de movilidad, en la práctica existen obstáculos que necesitan ser mapeados y requieren planes de trabajo efectivos.
En las experiencias estudiadas, utilizar datos de aplicaciones de movilidad para desarrollar iniciativas en el nivel estratégico significa un fuerte cambio de cultura institucional, que puede ser mejor estructurado partiendo de procesos que incluyan como pasos anteriores proyectos en los niveles operacional y táctico, como si fuera una "ruta segura" para construir compromiso y participación activa de los gestores de alto nivel jerárquico, como lo hicieron Río y Medellín.

En tal sentido, y para entender mejor las variaciones de esfuerzo en estos tipos de proyectos, seguidamente se propone una mensuración de los esfuerzos requeridos como producto del involucramiento de distintas instituciones en una iniciativa. Siguiendo la lógica de análisis propuesta, en la figura 10 los autores presentan las informaciones comentadas.

Como se observa en la figura 10, la intensidad de utilización de los recursos financieros, humanos y tecnológicos crece progresivamente del nivel operacional al estratégico. Lo mismo vale para la mayor participación de distintas agencias públicas en la medida en que se sube al nivel estratégico. En otras palabras, proyectos de nivel operacional presentan menor complejidad en cuanto a la utilización de recursos cuando se los compara con los niveles táctico y estratégico.

El nivel operacional permite ensayar las iniciativas de mayor potencial de impacto positivo en la ciudad, así como tener lecciones aprendidas suficientes para que sirvan de base para planear la iniciativa siguiente. Partiendo de potenciales entregas positivas de corto plazo, se generan un ambiente de confianza en el uso de datos y un marketing interno positivo en los gobiernos, lo que fomenta la construcción de una "ruta segura" $\gg$ Figura 10. Modelo evolutivo de la forma de uso de datos masivos por los gobiernos locales. Fuente: elaboración propia (2019).

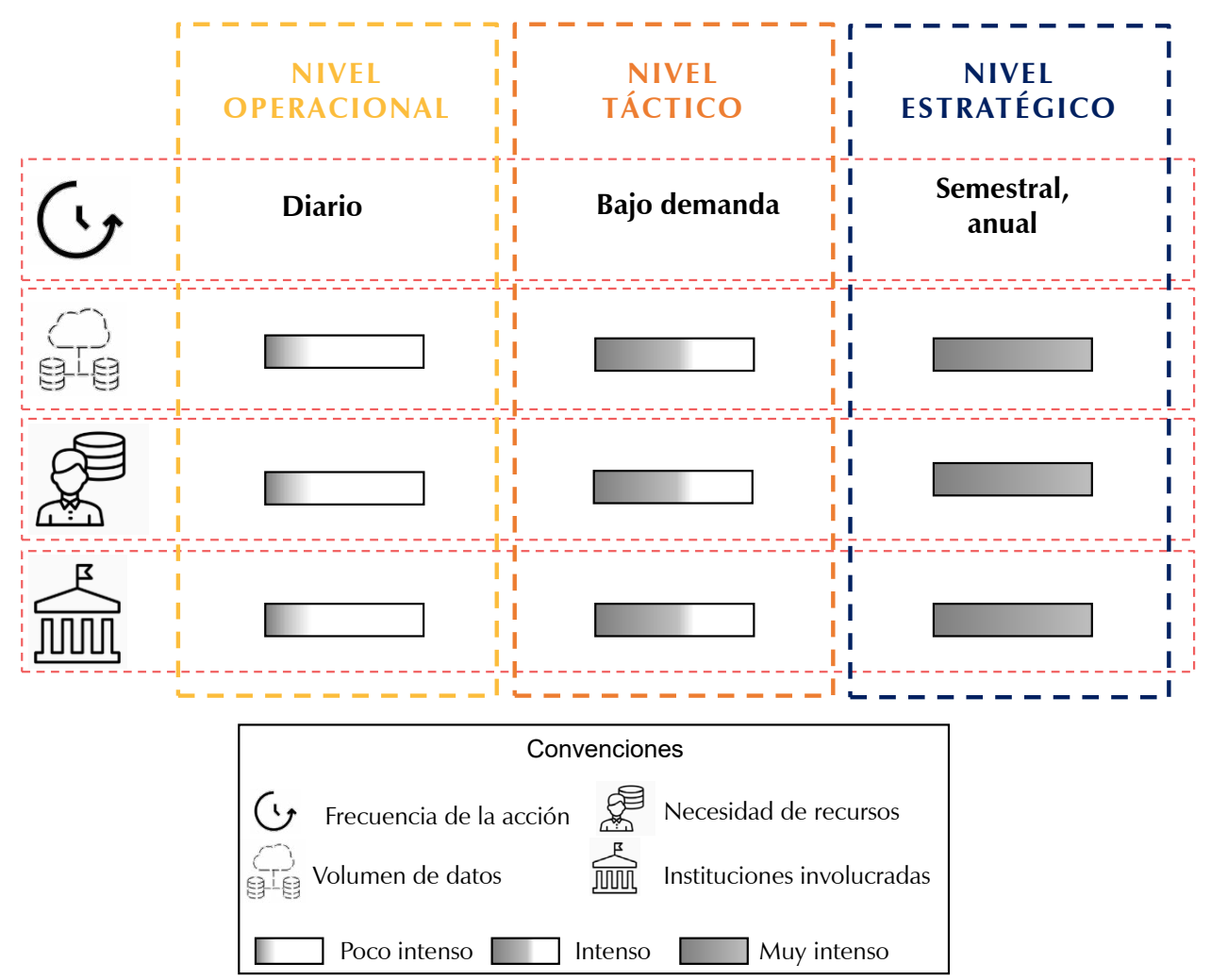


para el uso de datos, con planificación de proyectos más complejos de uso de datos para movilidad. Progresivamente, se alcanza a los profesionales de niveles jerárquicos más altos de las instituciones participantes y también es posible involucrar a aliados locales relevantes, como, por ejemplo, las universidades.

Para trazar la "ruta segura", es importante que los gobiernos sepan priorizar los proyectos que generen impactos claros en las rutinas de los ciudadanos, empezando por iniciativas que generen impactos positivos y de corto plazo. Tener claro dicho criterio desde el inicio de la alianza también es una estrategia relevante para obtener una validación y una participación más sólidas de las instituciones del gobierno y otros aliados.

Finalmente, la "ruta segura" tendrá más chance de ser exitosa si es capaz de centralizar las iniciativas de uso de datos masivos de movilidad; preferencialmente, en un centro de comando y control de tráfico; ello, porque los equipos de estos centros son los que más conocen los problemas de tráfico de la ciudad y los que más pueden beneficiarse de nuevas herramientas en sus rutinas. Dichos elementos fueron importantes, y, con seguridad, aportaron para el éxito del COR y del CIOS. Así, la temática de uso de datos por parte de los gobiernos empieza a ser vista de forma estratégica en estas y otras ciudades. En la medida en que los tomadores de decisión de las ciudades y de las empresas de apps perciben que su relación puede ser un gana-gana, ambos lados pasan a ser más flexibles y empiezan a compartir entre sí información y conocimiento sobre cómo utilizar los datos de manera colaborativa, y a crear así un ambiente más propicio para el desarrollo de nuevas (y más complejas) experiencias.

\section{Agradecimientos}

Los autores agradecen a Sergio Avelleda, exsecretario de movilidad de la ciudad de São Paulo, por la entrevista concedida.

\section{Listado de abreviaturas}

API Application Programming Interface (interfaz de programación de aplicaciones)

Apps aplicaciones móviles (acortamiento del inglés application)

BID Banco Interamericano de Desarrollo

CET-Rio Compañía de Ingeniería de Tráfico de la Alcaldía de Río de Janeiro

CIOS Centro de Inteligencia y Operación Semafórica de Medellín

COR entro de Operaciones de la Alcaldía de Río de Janeiro

DNP Departamento Nacional de Planeación de Colombia

IBGE Instituto Brasileño de Geografía e Estadística

SMT Secretaria Municipal de Mobilidade e Transportes de la Alcaldía de São Paulo

\section{Anexos}

\section{Anexo I. Encuesta aplicada a representantes de gobiernos locales}

1. Datos del respondiente:*

Nombre:

Ciudad:

Cargo:

2. En una escala de 0 a 5, donde 0 es "muy bajo", y 5, "muy alto", ¿cuál es el grado de interés de su ciudad en recibir los datos generados por las empresas de apps de servicios de movilidad urbana? *

$\bigcirc_{0} \bigcirc 1 \bigcirc 2 \bigcirc 3 \bigcirc 4{ }_{4} \bigcirc$

3. En una escala de importancia donde 0 es "poco relevante", y 5, "muy relevante", ¿cuáles son las motivaciones de su ciudad para acceder a los datos de las empresas de apps de servicios de movilidad urbana?

\begin{tabular}{|c|c|c|c|c|c|}
\hline & 1 & 2 & 3 & 4 & 5 \\
\hline \multicolumn{6}{|l|}{$\begin{array}{l}\text { Crear una buena relación con las empresas de apps } \\
\text { de movilidad urbana }\end{array}$} \\
\hline \multicolumn{6}{|l|}{$\begin{array}{l}\text { Utilizar los datos para formular, acompañar y monitorear } \\
\text { políticas públicas }\end{array}$} \\
\hline \multicolumn{6}{|l|}{$\begin{array}{l}\text { Utilizar los datos para apoyar la gestión operacional } \\
\text { de la movilidad urbana }\end{array}$} \\
\hline $\begin{array}{l}\text { Enviar y compartir datos de la ciudad en contrapartida } \\
\text { a los datos ofrecidos }\end{array}$ & & & & & \\
\hline
\end{tabular}


4. En una escala de importancia donde 0 es "poco relevante", y 5, "muy relevante", ¿̇cuáles son los principales retos para concretar alianzas con empresas de apps de servicios de movilidad urbana? *

Acuerdos jurídicos requeridos o normativas específicas del sector público

Incapacidad, por parte de la ciudad, para hacer contacto con las empresas de aplicaciones

Desconocimiento, por parte del Poder Público, de su ciudad, así como de los beneficios de la alianza planteada por la empresa

$\bigcirc \bigcirc \bigcirc \bigcirc \bigcirc$

Dificultad para convencer a las empresas de los beneficios de la alianza con la ciudad

Ausencia de voluntad política, por parte del actor público con poder de decisión, para establecer una alianza con la empresa

Ausencia de consenso acerca de las responsabilidades de inversión financiera que resultan de la alianza

5. Considerando el proceso de construcción de alianza entre su ciudad y una empresa de apps de servicios de movilidad urbana, en una escala de importancia donde 0 es "poco relevante", y 5, "muy relevante", ¿̇cuáles fueron las dificultades encontradas para, efectivamente, recibir los datos de la empresa? *

Ausencia de herramientas tecnológicas por parte de la ciudad

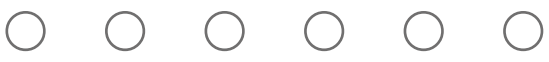

Ausencia de soluciones tecnológicas por parte de la empresa

Incompatibilidad entre las tecnologías adoptadas por la ciudad y la empresa

Carencia de personal calificado, por parte de la ciudad

Dificultades provenientes de la comunicación (manejo de otros idiomas diferencia horaria entre países, etc.)

Indisponibilidad de recursos financieros por parte de la ciudad para destinar a las actividades relacionadas con el compartimiento de datos

6. Considerando las alianzas existentes entre su ciudad y las empresas de apps de servicios de movilidad urbana, ¿su ciudad tuvo que hacer algún tipo de inversión para recibir los datos ofertados? Seleccione la(s) opción(es) que aplique(n). *

Recursos financieros para la adquisición de equipos o servicios específicos

Recursos humanos (personal)

Compra de activos físicos (mobiliario y equipos tecnológicos de bajo costo)

Compra de activos tecnológicos de grande porte o costo

Investimento financiero de grande porte para usos diversos

Otro (por favor, especifique):

\section{Anexo II. Encuesta aplicada a representantes de empresas}

1. Por favor, identifíquese: *

Nombre:

Empresa:

Cargo:

2. En una escala de importancia donde 0 es "poco relevante", y 5, "muy relevante", ¿cuáles son los objetivos de su empresa al abrir los datos para gobiernos locales? * 


Crear una buena relación con los gobiernos locales
Ofrecer datos para la formulación y el acompañamiento de políticas
públicas
Ofrecer herramientas y apoyar la gestión operacional de la movilidad
urbana en las ciudades
Recibir datos de la ciudad en contrapartida de los datos ofrecidos

3. En una escala de importancia donde 0 es "poco relevante", y 5, "muy relevante", ¿̇cuáles son los principales retos para concretar alianzas con gobiernos locales?

Acuerdos jurídicos requeridos o normativas específicas del sector público
Incapacidad, por parte de la empresa, para hacer contacto con los actores
tomadores de decisiones (de los gobiernos locales) en cuanto a acuerdos
Dificultad para convencer a los gobiernos locales de los beneficios de la
alianza planteada por la empresa
Ausencia de voluntad política, por parte del actor público con poder de
decisión, para establecer una alianza con la empresa
Ausencia de consenso acerca de las responsabilidades de inversión
financiera que resultan de la alianza

4. Considerando los casos en que su empresa logró hacer una alianza con el gobierno local, en una escala de importancia donde 0 es "poco relevante", y 5, "muy relevante", ¿cuáles fueron las dificultades encontradas para, efectivamente, compartir datos con los gobiernos locales?

Ausencia de herramientas tecnológicas por parte del gobierno (hardware)
Ausencia de soluciones tecnológicas por parte de la empresa (software)
Incompatibilidad entre las tecnologías adoptadas por el gobierno local y la
empresa
Carencia de personal calificado, por parte de los gobiernos
dificultades provencia horaria entre países, etc.)
Indisponibilidad de recursos financieros por parte del gobierno local para
destinar a las actividades relacionadas con el compartimiento de datos

\section{Referencias}

Avelleda, S. (2018, abril 4). Congreso Internacional Movilidad y Transporte. Recuperado de: https://feriamovilidadytransporte.com/ memo/a/1/cf1/sergio-avelleda.pdf

Avelleda, S. (2018). Prácticas innovadoras en la planeación del transporte. Bogotá, Colombia. Recuperado de:

https://drive.google.com/drive/ folders/1JwmxniXw8zohOyyMAuru1tDXFSaQCKv

Azzone, G. (2018). Big data and public policies: Opportunities and challenges. Statistics and Probability Letters, 116-120. https://doi.org/10.1016/j.spl.2018.02.022

Carranza, M., Montoya, F. G., Valdés, A., González, C., Jaramillo, M., \& Henao, S. (2018). Asignación dinámica de planes semafóricos como estrategia de descongestión vehicular. Medellín. Recuperado de: https://minas.medellin.unal.edu.co/ gruposdeinvestigacion/gaunal/images/ imagenes/Eventos/MOVICI_MOYCOT/ sesion3/4-Mauricio-Carranza.pdf

Decreto, № 58.595 (Prefeitura do Municipio de Sao Paulo 4 de Janeiro de 2019).
DNP. (2019, septiembre). Terridata. Recuperado de: https://terridata.dnp.gov.co/index-app. html\#/perfiles/05001

ITDP Brasil. (2019). Uso de dados e evidências para planejamento e gestão da mobilidade urbana Oficina de troca de experiências. Rio de Janeiro: ITDP Brasil. Recuperado de: http://itdpbrasil.org/wp-content/uploads/2019/04/ ITDP_Oficina-MobiliDADOS-2019_v03.pdf

Manyika, J., Chui, M., Brown, B., Bughin, J., Dobbs, R., Roxburgh, C., \& Byers, A. H. (2011). Big data: The next frontier for innovation, competition, and productivity. McKinsey Global Institute. Recuperado de:

https://www.mckinsey.com/ /media/ McKinsey/Business\%20Functions/ McKinsey\%20Digital/Our\%20lnsights/Big\%20 data $\% 20$ The $\% 20$ next $\% 20$ frontier $\% 20$ for $\% 20$ innovation/MGI_big_data_exec_summary.ashx

Mónico, L. S., Alferes, V. R., Castro, P. A., \& Parreira, P. M. (2017). A observação participante enquanto metodología de investigação qualitativa. $6^{\text {a }}$ Congreo Ibero-Americano en Investigación Cualitativa, Vol. 3, (pp. 724-733). Recuperado de: https://proceedings.ciaiq.org/index.php/ ciaiq2017/article/download/1447/1404/
Osorio Arjona, J., \& García Palomares, J. C. (2017). Nuevas fuentes y retos para el estudio de la movilidad urbana. Cuadernos Geográficos; 56(3), 247-267. Recuperado de:

https://revistaseug.ugr.es/index.php/cuadgeo/ article/view/5352

Rodríguez, P. (2013 diciembre 16). Smart Mobility: movilidad inteligente en las ciudades. Valencia: Universidad Politecnica de Valencia. Recuperado de:

http://www.i-ambiente.es/?q = blogs/smartmobility-movilidad-inteligente-en-lasciudades

Sandí Meza, V. (2017, junio). Las alianzas público-privadas como elemento de gestión de las ciudades inteligentes. ICAP-Revista Centroamericana de Administración Pública (72), 15-31.

https://doi.org/10.35485/rcap72_1

Vargas, G. T., \& Sánchez, J. A. (2002). Métodos de asignación de tránsito en redes regionales de carreteras: dos alternativas de solución. IMT Instituto Mexicano de Transporte, 214, 7-99. Recuperado de: http://worldcat.org/issn/01887297 


\section{Vol.}
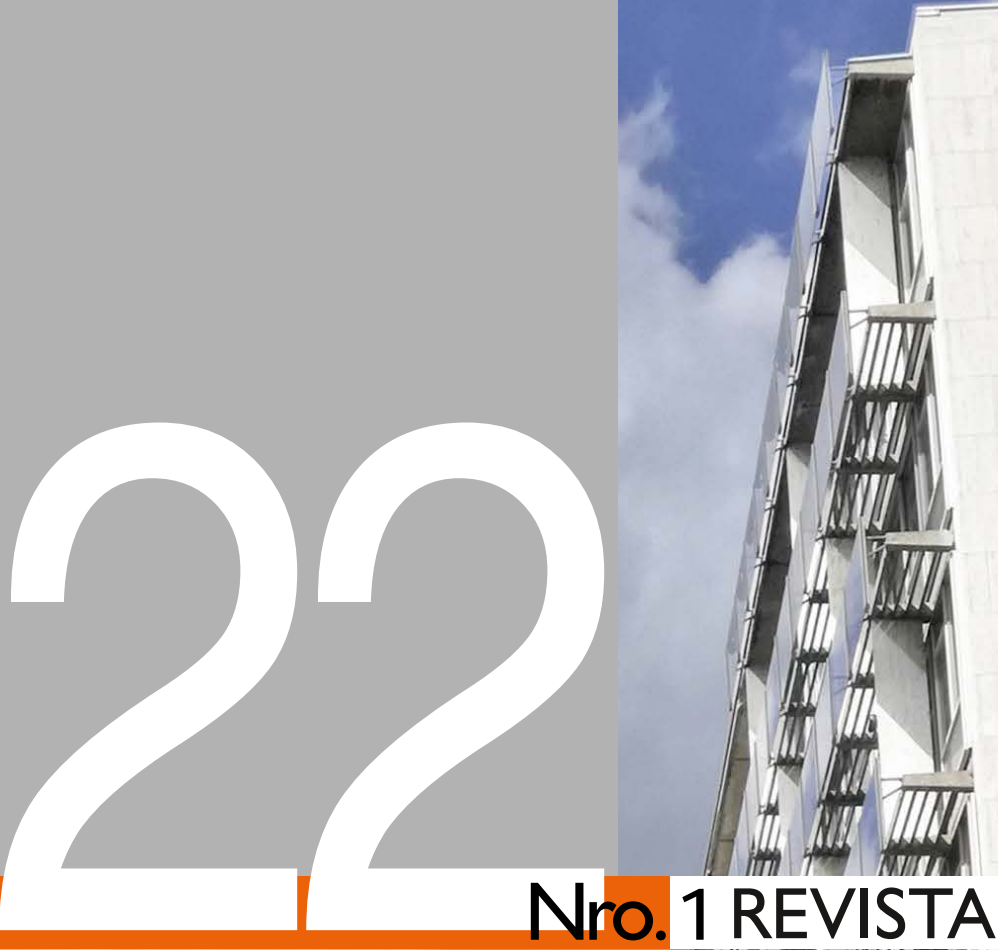

ISSN: 1657-0308 (Impresa)

Nro. 1 REVISTA DE ARQUITECTURA

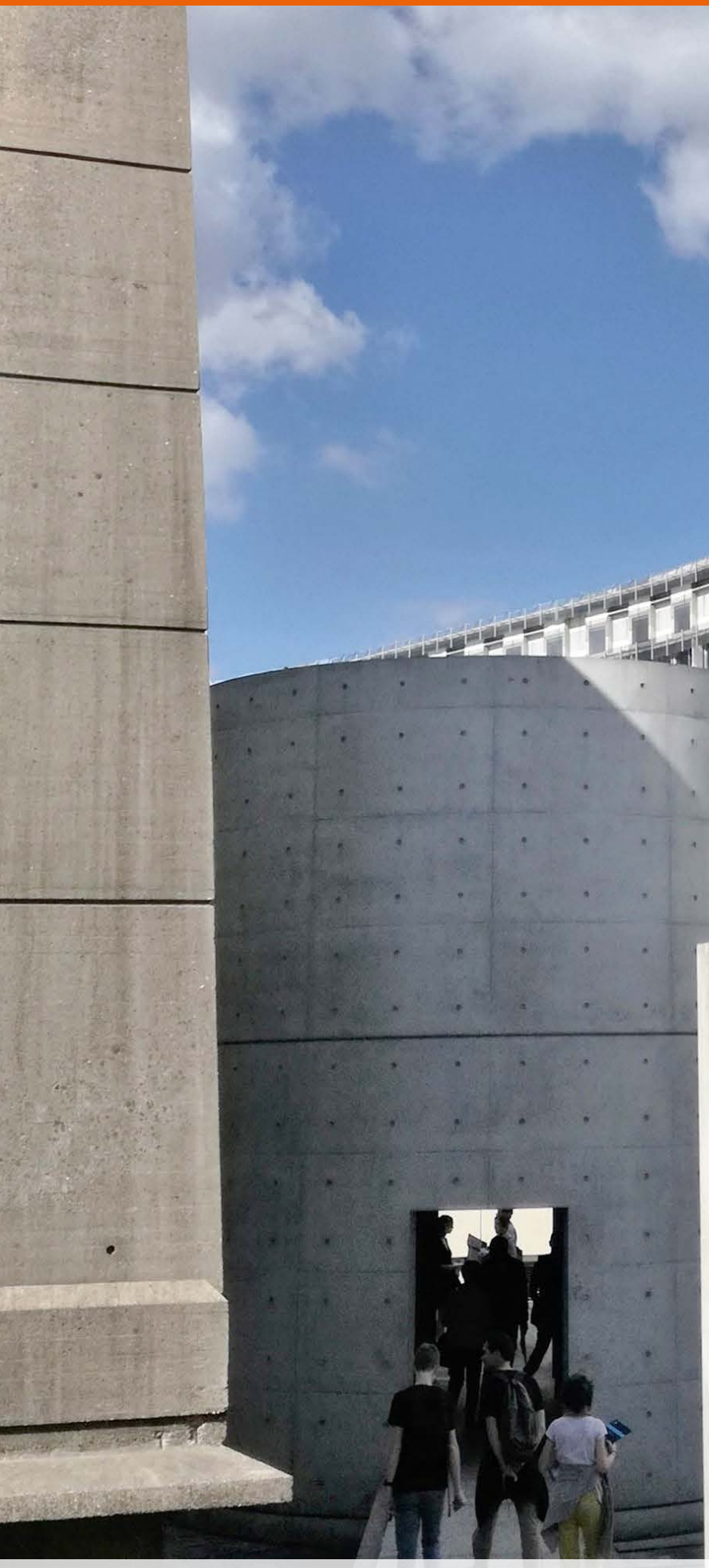

$\overline{9}$
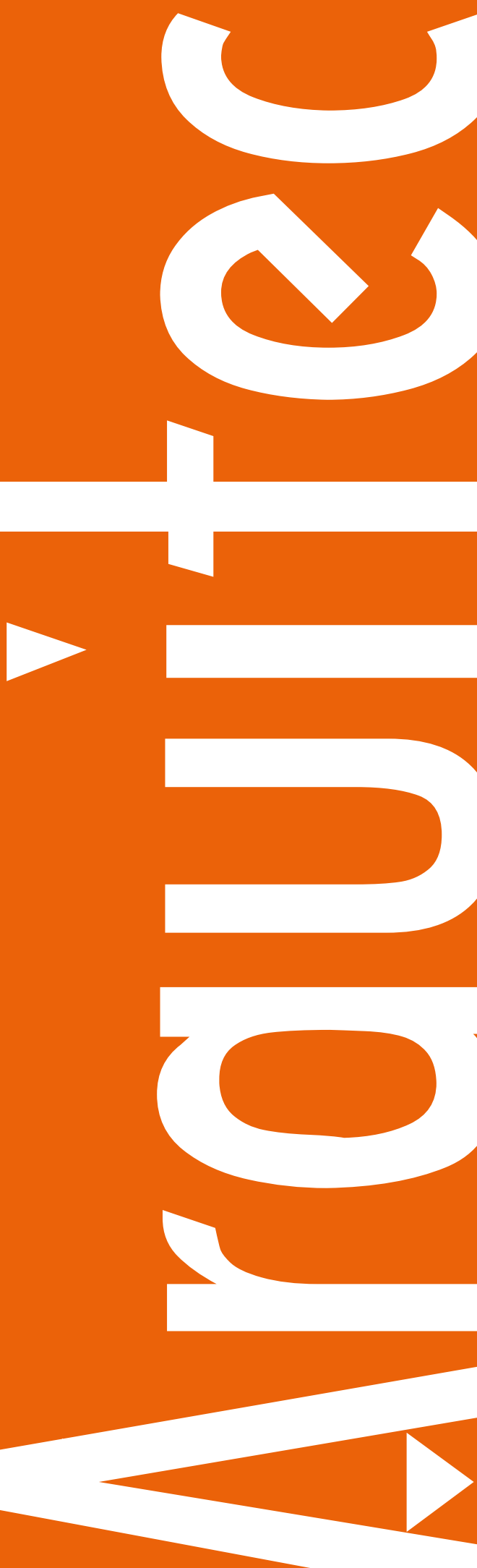


\section{Enfoque y alcance}

La Revista de Arquitectura (Bogotá) ( (ISSN 1657-0308 Impresa y E-ISSN 2357-626X en línea) es una publicación científica seriada de acceso abierto, arbitrada mediante revisión por pares (doble ciego) e indexada, en donde se publican resultados de investigación originales e inéditos.

Está dirigida a la comunidad académica y profesional de las áreas afines a la disciplina. Es editada por la Facultad de Diseño y el Centro de Investigaciones (CIFAR) de la Universidad Católica de Colombia en Bogotá (Colombia).

La principal área científica a la que se adscribe la Revisto de Arquitectura (Bogotá) según la OCDE es:

Gran área: 6. Humanidades

Área: 6.D. Arte

Disciplina: 6D07. Arquitectura y Urbanismo

También se publican artículos de las disciplinas como 2A02, Ingeniería arquitectónica; 5C03, Estudios urbanos (planificación y desarrollo); 6D07, Diseño.

Los objetivos de la Revista de Arquitectura (Bogotá) son:

- Promover la divulgación y difusión del conocimiento generado a nivel local, nacional e internacional

- Conformar un espacio para la construcción de comunidades académicas y la discusión en torno a las secciones definidas.

- Fomentar la diversidad institucional y geográfica de los autores que participan en la publicación.

- Potenciar la discusión de experiencias e intercambios científicos entre investigadores y profesionales.

- Contribuir a la visión integral de la arquitectura, por medio de la concurrencia y articulación de las secciones mediante la publicación de artículos de calidad.

- Publicar artículos originales e inéditos que han pasado por revisión de pares, para asegurar que se cumplen las normas éticas, de calidad, validez científica, editorial e investigativa.

- Fomentar la divulgación de las investigaciones y actividades desarrolladas en la Universidad Católica de Colombia.
Palabras clave de la Revista de Arquitectura (Bogotá): arquitectura, diseño, educación arquitectónica, proyecto y construcción, urbanismo.

Idiomas de publicación: español, inglés, portugués y francés. Título abreviado: Rev. Arquit.

\section{Titulo corto: RevArq}

\section{Políticas de sección}

La revista se estructura en tres secciones correspondientes a las líneas de investigación activas y aprobadas por la institución, y dos complementarias, que presentan dinámicas propias de la Facultad de Diseño y las publicaciones relacionadas con la disciplina.

Cultura y espacio urbano. En esta sección se publican los artículos que se refieren a fenómenos sociales en relación con el espacio urbano, atendiendo aspectos de la historia, el patrimonio cultural y físico, y la estructura formal de las ciudades y el territorio.

Proyecto arquitectónico y urbano. En esta sección se presentan artículos sobre el concepto de proyecto, entendido como elemento que define y orienta las condiciones proyectuales que devienen en los hechos arquitectónicos o urbanos, y la forma como estos se convierten en un proceso de investigación y nuevo de conocimiento. También se presentan proyectos que sean resultados de investigación, los cuales se validan por medio de la ejecución y transformación en obra construida del proceso investigativo. También se contempla la publicación de investigaciones relacionadas con la pedagogía y didáctica de la arquitectura, el urbanismo y el diseño.

Tecnología, medioambiente y sostenibilidad. En esta sección se presentan artículos acerca de sistemas estructurales, materiales y procesos constructivos, medioambiente y gestión, relacionados con los entornos social-cultural, ecológico y económico.

Desde la Facultad. En esta sección se publican artículos generados en la Facultad de Diseño, relacionados con las actividades de docencia, extensión, formación en investigación o internacionalización, las cuales son reflejo de la dinámica y de las actividades realizadas por docentes, estudiantes y egresados; esta sección no puede superar el $20 \%$ del contenido.

Textos. En esta sección se publican reseñas, traducciones y memorias de eventos relacionados con las publicaciones en Arquitectura y Urbanismo.
A Frecuencia de publicación

Desde 1999 y hasta el 2015, la Revista de Arquitectura (Bogotá) publicó un volumen al año, a partir del 2016 se publicarán dos números por año en periodo anticipado, enero-junio y julio-diciembre, pero también maneja la publicación anticipada en línea de los artículos aceptados (versión Post-print del autor).

La Revista de Arquitectura (Bogotá) se divulga mediante versiones digitales (PDF, HTML, EPUB, XML) e impresascon un tiraje de 700 ejemplares, los tiempos de producción de estas versiones dependerán de los cronogramas establecidos por la editorial.

Los tiempos de recepción-revisión-aceptación pueden tardar entre seis y doce meses dependiendo del flujo editorial de cada sección y del proceso de revisión y edición adelantado.

Con el usuario y contraseña asignados, los autores pueden ingresar a la plataforma de gestión editorial y verificar el estado de revisión, edición o publicación del artículo.

\section{A Canje}

La Revista de Arquitectura (Bogotá) está interesada en establecer canje con publicaciones académicas, profesionales o científicas del área de Arquitectura y Urbanismo, como medio de reconocimiento y discusión de la producción científica en el campo de acción de la publicación.

\section{Mecanismo}

Para establecer canje por favor descargar, diligenciar y enviar el formato: RevArq FP20 Canjes
Universidad Católica de Colombia (2020, enero-junio). Revista de Arquitectura (Bogotá), 22(I) I-188. Doi: 10.14718

ISSN: 1657-0308 E-ISSN: 2357-626X

Especificaciones:

Formato: $34 \times 24 \mathrm{~cm}$

Papel: Mate $115 \mathrm{~g}$

Tintas: Negro y policromía
A Contacto

Dirección postal:

Avenida Caracas No. 46-72.

Universidad Católica de Colombia

Bogotá D.C.(Colombia)

Código postal: 111311

Facultad de Diseño Centro de Investigaciones (CIFAR). Sede El Claustro. Bloque " $\mathrm{L}$ ", 4 piso Diag. 46A No. $15 \mathrm{~b}-10$ Editor, Arq. César Eligio-Triana

Teléfonos:

+57 (1) $3277300-3277333$

Ext. 3109; 3112 o 5146

Fax: +57 (1) 2858895
Correo electrónico: revistadearquitectura@ucatolica.edu.co cifar@ucatolica.edu.co

Página WEB: www.ucatolica.edu.co vínculo Revistas científicas

http://publicaciones.ucatolica.edu.co revistas-cientificas http://editorial.ucatolica.edu.co/ojsucatolica/revistas ucatolica/index.php/RevArq 
